\title{
A Novel Parametric Model for Nonlinear Hysteretic Behaviors with Strain Stiffening of Magnetorheological Gel Based on Fruit Fly Optimization Algorithm
}

\section{Guang Zhang ( $\nabla$ xzjx092@163.com )}

Zhejiang University of Technology https://orcid.org/0000-0002-5292-3857

\section{Zheng Zhang}

Zhejiang University of Technology

\section{Min Sun}

Zhejiang University of Technology

\section{Yang Yu}

University of Technology Sydney

Jiong Wang

Nanjing University of Science and Technology

Shibo Cai

Zhejiang University of Technology

\section{Research Article}

Keywords: Magnetorheological gel, parametric model, strain stiffening, nonlinear hysteretic loop, fruit fly optimization algorithm, parameter generalization

Posted Date: December 21st, 2021

DOI: https://doi.org/10.21203/rs.3.rs-1136911/v1

License: (c) (1) This work is licensed under a Creative Commons Attribution 4.0 International License. Read Full License 


\section{Abstract}

Magnetorheological (MR) gel is a new branch of MR materials, which can overcome the phenomenon of particle agglomeration existing in MR fluid, thus improving the controllability of materials in engineering applications. In this paper, a novel parametric model for tracking the nonlinear hysteretic behaviors with strain stiffening of MR gel is constructed. The measure data in relative to the five current levels of $0 \mathrm{~A}$, $0.2 \mathrm{~A}, 0.5 \mathrm{~A}, 0.8 \mathrm{~A}$ and $1 \mathrm{~A}$ under the strain amplitude and frequency of $10 \%$ and $0.1 \mathrm{~Hz}$ respectively are utilized to identify the parameters. The optimal solution for the model parameters is conduced employing the fruit fly optimization algorithm (FOA). The comparison study with two typical model such as BoucWen model and viscoelastic-plastic model is conduced to to evaluate the effectiveness of the developed model. The model parameters are generalized with respect to the loading current, and the reliability of the generalized model is verified. The studies show that the proposed model can perfectly fit the strain stiffening and nonlinearity of sample, which can provide a theoretical basis for the semi-active control of MR gel in practical engineering applications.

\section{Introduction}

Magnetorheological (MR) gel as a new brand of MR materials, which is a kind of colloidal suspensions that reveals extraordinary reversible variety in viscoelastic behaviors when subjected upon by extra magnetic field [1]. In the MR gel system, with the micro-sized soft magnetic particles dispersed in the polymer matrix uniformly without magnetic excitation and formed tens of thousands of chain-like structures along the direction of the magnetic field instantaneous, which shows continuous changes in mechanical properties. The most remarkable variety in the mechanical properties of the MR gel along with magnetic field is its viscoelastic characteristics [2-4]. In addition, the inherent virtue of MR gel is that it can overcome the phenomenon of particle agglomeration existing in MR fluid, thus improving the controllability of materials [5]. These unique performances allow MR gel have great potential in engineering application, such as damping tunable damper, torque controlled clutch, self-adjusting polishing device, achieving semi-active control.

The crucial role of the MR gel is to change its viscoelastic properties by using magnetic field to meet the requirements of the actual engineering application. For instance, by identifying the recoil force characteristics of gun in different periods and analyzing the mechanical responses, the viscoelastic behaviors of the MR gel should be adjust to achieve the goal of shooting continuously and accurately [6]. In order to better use of the unique controllable characteristics of MR gel, a computational-efficient and reliable model should be constructed for developing the corresponding semi-active control system. Until now, some efforts have been made to achieve this purpose [7-10], there is still lots of difficulties and problem due to the description of the nonlinear hysteretic characteristics and strain stiffening behaviors of the MR gel is a challenging work.

Up to present the model has been developed for describing the unique properties of the MR materials/devices under dynamic condition, which is commonly divided into two categories: 
nonparametric and parametric models [11]. Generally speaking, nonparametric model need to employ the artificial intelligence technology such as support vector machine (SVM) and neural network in the process of building the model to predict the nonlinear hysteresis responses of the MR materials/devices with high precision. Therefore, nonparametric model can fit many different function forms with very excellently prediction performance even there are no or minor assumptions about the objective function. Yu et al developed an artificial neural network based nonparametric model with one output neuron, three input neurons and eight hidden neurons to capture the nonlinear hysteretic behaviors of MR elastomer based isolator. The training result proves that the superiority and effectiveness of the constructed model in predicting MR elastomer based isolator [12]. However, the prediction process consume long time due to the requirement of the enormous training data and there are indeterminacy physical explanation for the nonparametric model.

The parametric model has the ability to overcome inherent defect attaching with nonparametric model with high efficiency and precision and every model parameter corresponding to a special physical explanation. Among them, Bouc-Wen model is common-used parametric model with eight parameters, which is firstly proposed by Bouc and ameliorated by Wen $[13,14]$. Several studies prove that the BoucWen model has ability to portray nonlinear hysteresis responses with extraordinary performance. However, it failure to capture region where opposite signs of the velocity and acceleration. To precise pursue nonlinear hysteretic properties especial for the position where opposite signs of the velocity and acceleration, Bouc-Wen has been modified by Spencer et al and ten model parameters are generated, which is called as modified Bouc-Wen model [15]. However, the number of the parameter for above models is large, which result in the identification of the parameter becomes academically complexly and increases the computational memory meanwhile. Therefore, Kamath et al [16] have proposed a viscoelastic-plastic model only with six parameters to present the nonlinear dynamic behaviors of ER material. The viscoelastic-plastic model is proposed considering that there are three periods during shear process of the ER material, i.e., pre-yield stage, yield stage and post-yield stage, which is composed of preyield stress, yield stress and post-yield stress connecting in parallel. The time required for parameter identification is greatly shortened by employing viscoelastic-plastic model.

Another important factor related to the computational efficient is the selection of the optimization algorithm. Until now, there are several kinds of optimization algorithm are employed to achieve the identification of the parameters such as fruit fly optimization algorithm (FOA) [17], particle swarm optimization (PSO) [18] and genetic algorithm (GA) [19]. The root means square error (RMSE) between experimental values and predict results as fitness function is utilized to identify model parameters. Among them, FOA is a novel swarm optimization algorithm that can arrive at the global optimal result extraordinary fast with an excellent convergence property compared with other algorithms due to the fruit fly itself is superior to other species in sensory perception, especially in smell and vision [20]. Yu et al utilized the self-adaptive step FOA to portray the hysteresis responses of the MR elastomer based isolator and the result shown that FOA has an excellent performance to identify the model parameters with rapid convergence [21]. 
Therefore, in this paper, a novel computationally efficient model with less parameters is proposed to capture the nonlinear hysteretic behaviors and strain stiffening of the MR gel. This model employs a tangent function related to the shear strain combined with a dashpot and hysteresis operator to describe the hysteretic properties. The FOA is utilized to identify the model parameter due to its fast convergence. The hysteretic responses of the MR gel under five levels of the currents, i.e., $0 \mathrm{~A}, 0.2 \mathrm{~A}, 0.5 \mathrm{~A}, 0.8 \mathrm{~A}$ and $1 \mathrm{~A}$ with the shear strain and shear frequency are $10 \%$ and $0.1 \mathrm{~Hz}$ are employed to testify the efficient and feasibility of the constructed model. The Bouc-Wen model and the viscoelastic-plastic model are utilized as comparison group.

\section{$2 \mathrm{Mr}$ Gel And Measurement}

Polyurethane based MR gel with $70 \mathrm{wt} \%$ content of carbonyl iron particles (CIP) is prepared according to the reference 8 , which is shown in Fig. 1 a. To prove the stability and reliability of self-developed MR gel, the microstructure is studied using a scanning electron microscope (SEM, model: 250FEG) and the result is exhibited in Fig. 1b. The white circle and black background represent CIP and polyurethane based matrix respectively from Fig. $1 \mathrm{~b}$, which shows that the CIPs are uniformly dispersed in the matrix. Therefore, the developed MR gel shows the characteristics of stability and reliability. The magnetic physical properties of the MR gel is studied by employed the vibrating sample magnetometer (VSM, model: Lake Shore 7407) and the result is shown in Fig. 1c. it can be concluded from Fig. 1c that the magnetic flux density of the CIP is higher than that of the MR gel in the same magnetic field. This phenomenon indicates that the magnetic field sensitivity decreases with the addition of matrix.

The MR material based devices are commonly used in the dynamic shear condition, which indicates that the dynamic responses of the MR gel is very important to achieve the feasibility of the control in engineering application. Therefore, the large-amplitude-oscillation-stress (LAOS) measurements under five levels of the currents, i.e., $0 \mathrm{~A}, 0.2 \mathrm{~A}, 0.5 \mathrm{~A}, 0.8 \mathrm{~A}$ and $1 \mathrm{~A}$ are conducted using MRC302 at room temperature $\left(20^{\circ} \mathrm{C}\right)$, which is exhibited in detail in Fig. 2a. During the process of the test, the strain amplitude and frequency are set as $10 \%$ and $0.1 \mathrm{~Hz}$ respectively, which is shown in Fig. $2 \mathrm{~b}$. The dynamic response of the MR gel is shown in Fig. 2c. It indicated from Fig. 2c that the dynamic response curve under all current shows viscoelastic and hysteretic characteristics and the maximum stress increases along with current excitation. It worth to note that the hysteresis response corresponding to the zero magnetic field shows approximately linear characteristics. However, it exhibits obvious nonlinear properties under the application of magnetic field and the strain stiffening phenomenon appear, which is shown in detail in Fig. 3.

Figure 3 shows the hysteresis response of the sample under the current of $1 \mathrm{~A}$. It is clearly observed that there are two strain stiffening effects in one cycle, which are expressed in the elliptic region. The interpretation of the appearance of strain stiffening is mainly due to the fact that the polymer chain of the polyurethane has extraordinary constrained ductility for the CIP [22]. In addition, the interaction force between two adjacent particles affects the motion of particles, which further hinders the movement of the polymer chain. Therefore, in Fig. 3 , the strain stiffening phenomenon appear at the point where line $L_{1}$ 
and $L_{2}$ start to far away from the hysteresis loop, which are marked as $S_{1}$ and $S_{2}$. In a word, the nonlinear viscoelastic behaviors and strain stiffening characteristics of the ME gel is significantly complicated, which is related to the loading current. For achieve the semi-active of the MR gel in the engineering application, the crucial and meaningful task is to develop a perfect parametric model to accurately describe such complex nonlinear hysteretic characteristics with strain stiffening exciting in MR gel.

\section{Modeling Of The Mr Gel}

\subsection{Classical Bouc-Wen model}

Recently, lots of parameter model is constructed to capture the nonlinear and hysteretic characteristics of the MR material and device. Among them, Bouc-Wen model is common-used phenomenological model [2, 23], which is composed of viscous dashpot, spring and hysteresis operator connecting in parallel showing in Fig. 4. The stress/force excited by Bouc-Wen model can be described as following:

$$
{ }^{p r e}=\alpha z+k_{1}\left(\gamma-\gamma_{0}\right)+c_{1} \dot{\gamma}
$$

1

$$
\dot{\mathrm{z}}=\mathrm{A} \dot{\gamma}-\beta \dot{\gamma}|z|^{n}-\gamma|\dot{\gamma}| z|z|^{n-1}
$$

In which $\gamma$ and $\dot{\gamma}$ are strain and rate, respectively; $k_{1}$ and $c_{1}$ are elastic coefficient and viscous coefficients; $x_{0}$ is initial deformation of spring and $\mathrm{z}$ is an operator and $\dot{z}$ is the time derivative of $z$, the parameters of $\mathrm{A}, \beta$ and $\gamma$ and $n$ are coefficients of the model. Therefore, $A$ series of eight parameters should be determined to capture the hysteretic behaviors as shown in the followings:

$$
\exists=\left[A, \beta, \gamma, n, \alpha, k_{1}, c_{1}, x_{0}\right]
$$

\subsection{Viscoelastic-plastic Model}

The viscoelastic-plastic model is proposed considering that there are three periods during shear process of the MR material, i.e., pre-yield stage, yield stage and post-yield stage $[4,24]$. The principle of the viscoelastic-plastic model is presented in Fig. 5, which is composed of pre-yield stress, yield stress and post-yield stress connecting in parallel. Therefore, the shear stress generated by viscoelastic-plastic model can be expressed as following:

$$
{ }^{\text {pre }}=k_{e}(\dot{\gamma}) \tau_{e}(\gamma, \dot{\gamma})+k_{i}(\dot{\gamma}) \tau_{i}(\dot{\gamma})+k_{y}(\dot{\gamma}) \vartheta_{y}
$$


Where $k_{e}(\dot{\gamma}), k_{i}(\dot{\gamma})$ and $k_{y}(\dot{\gamma})$ are hysteresis operators, which can be obtained by Eqs (4), (5) and (6) respectively; $\tau_{e}(\gamma, \dot{\gamma})$ and $\tau_{i}(\dot{\gamma})$ are viscoelastic induced stress, which expressed by Eqs (7) and (8).

$$
k_{e}(\dot{\gamma})=\frac{1}{2}\left(1-\tanh \left(\frac{|\dot{\gamma}|-\rho}{4 \omega}\right)\right)
$$

4

$$
k_{i}(\dot{\gamma})=\frac{1}{2}\left(1+\tanh \left(\frac{|\dot{\gamma}|-\rho}{4 \omega}\right)\right)
$$

5

$$
k_{y}(\dot{\gamma})=\frac{1}{2} \tanh \left(\frac{\dot{\gamma}}{4 \omega}\right)
$$

6

$$
\tau_{e}(\gamma, \dot{\gamma})=\left(k_{1} \gamma+c_{1} \dot{\gamma}\right)
$$

7

$$
\tau_{i}(\dot{\gamma})=c_{2} \dot{\gamma}
$$

8

In which $k_{1}$ is elastic coefficient; $c_{1}$ and $c_{2}$ are viscous coefficients. In a word, there are six parameters should be identified to predict the hysteretic behaviors accuracy as following:

$$
\exists=\left[\rho, \omega, k_{1}, c_{1}, c_{2}, \vartheta_{y}\right]
$$

\subsection{Proposed Novel Parametric Model}

As described in section 2, the dynamic hysteresis loop under test condition of the sample behaviors two main properties, i.e., nonlinear viscoelastic hysteretic characteristics and obvious strain stiffening phenomenon. Among them, hysteretic properties can be capture by a differential operator. The viscous 
behaviors is relative to shear rate, which can be featured by a dashpot. The elastic characteristics and strain stiffening phenomenon are both bound up with strain. Consequently, the elastic characteristics can be described by a spring and the strain stiffening phenomenon is similar to tangent function, which is denoted from Fig. 6 . Therefore, the novel parametric model is composed of a dashpot, a hysteresis operator and a spring relating to tangent function, which is shown in Fig. 7. The stress generated by the proposed model can be expressed as following:

$$
{ }^{\text {pre }}=\alpha \mu+\mathrm{c} \dot{\gamma}+k \tan (\gamma)
$$

9

In which $\tau^{\text {pre }}, \gamma$ and $j$ are the modeling stress, shear strain, and shear rate respectively; $\mathrm{c}$ and $k$ present viscosity and elasticity coefficient; $\alpha$ is the hysteresis operator coefficient; $\mu$ is a hysteresis differential operator, which is denoted as following:

$$
\dot{\mu}=\delta \dot{\gamma}-\epsilon|\dot{\gamma}| \mu|\mu|-\beta \gamma
$$

10

Where $\backslash \operatorname{dot}\{\{\backslash \mathrm{mu}\}\}$ is the time derivative of $\{\backslash \mathrm{mu}\} ;$; delta, $\epsilon$, and $\backslash$ beta are the parameters for the operator. Therefore, there are six parameters should be identified to accuracy capture the experimental result as list in follows:

lexists $=\backslash$ eft $[\{\backslash$ alpha $\}, \backslash$ text $\{c\}, \backslash$ text $\{k\}, \backslash$ delta $, €, \backslash$ beta $\backslash$ right $]$

In this work, the mid-various, i.e., Imu in Equ(10) is solved by the Euler method due to the differential form. The employment of the Euler formula to obtain the mid-parameter $\mathrm{z}$ is described as following:

$\{\backslash m u\}_{-}\{i+1\}=\{\backslash m u\}_{-}\{i\}+\backslash$ varDelta $t \backslash$ bullet $\backslash \operatorname{dot}\left\{\{\backslash m u\}_{-}\{i\}\right\}+o \backslash$ left $\left(\{\backslash \text { varDelta } t\}^{\wedge}\{2\} \backslash\right.$ right $)$

11

Where IvarDelta $t$ is the time interval between the two connected data points.

\section{Problem Description And Modeling}

\section{1 problem statement}

After the parametric model is constructed based on the hysteretic behaviors of the MR gel. The following task is to identify the model parameters by capturing the stress-strain and the stress-rate responses under all loading excitation. According to the dynamic hysteresis responds of the MR gel from Fig. 3, showing two obvious characteristics of strain stiffening and nonlinear viscoelasticity. Therefore, the process of the identification of the model parameters is difficult to achieve by trials. The minimization optimization method is a perfect way to solve the problem. Then the critical point is the contracture of the fitness 
function Fit\left(\{lexists \}_\{i\}\right), which has an crucial impact on the identification of the model parameters. In those operations, the root means square error (RMSE) between testing result and model predicting value is employed as the fitness function, which can be expressed as following:

fit \left } ( \backslash \text { exists } \backslash \text { right } ) = \backslash \operatorname { t e x t } \{ R \} \backslash \operatorname { t e x t } \{ M \} \backslash \operatorname { t e x t } \{ S \} \backslash \text { text } \{ E \} \backslash \text { left } ( \backslash \text { exists } \backslash \text { right } ) = \backslash \operatorname { s q r t } \{ \backslash \text { frac } \{ 1 \} \{ N \} \backslash \text { sum _ } \{ i = 1 \} ^ { \wedge } \{ N \}

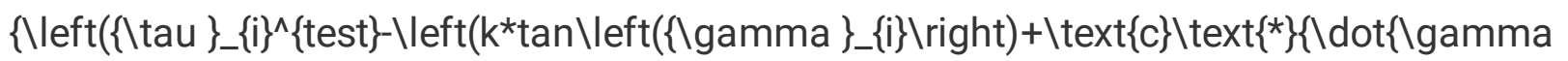
\}$\}_{-}\{i\}+\backslash$ text $\{\epsilon\} \backslash \operatorname{text}\left\{{ }^{\star}\right\}\{\backslash \mathrm{mu}\}_{-}\{i\} \backslash$ right $) \backslash$ right $\left.\left.)\right\}^{\wedge}\{2\}\right\}$ (12) Where $\backslash$ exists is the parameter set of the proposed model; $N$ is the overall number of testing data; $\{\backslash \operatorname{tau}\} \_\{j\}^{\wedge}\{\exp \}$ is the test stress result. If the value of the fit \left(\exists \right) is close to the zero, the corresponding value of the \exists can be employed as the optimal solution. In other words, the lower the value of fit \left(\exists \right), the better the parameters are obtained. Generally speaking, the minimax optimal event with necessary constraint condition can be described in detail as following:

$\backslash$ text $\{m\} \backslash$ text $\{i\} \backslash$ text $\{n\} F i t \backslash$ eft( $($ exists $\backslash$ right $)$ s.t. $\{c\}_{-}\{0\}>0,\{k\} \_\{0\}>0$ 13

The FOA is employed to solve the minimax problem due to the fruit fly itself is superior to other species in sensory perception, especially in smell and vision, which is shown in Fig. 5. The FOA can arrive at the global optimal result extraordinary fast with an excellent convergence property compared with other commonly used swarm algorithms $[20,25]$. The process of the FOA to achieve the optimal parameter set of the constructed model for MR gel can be composed of the following steps:

1. Read the test data such as shear stress and shear strain and calculate the shear rate;

2. Definition of the optimization problem and set the FOA algorithm parameters, i.e., population size $\{N\}_{-}\{p\}=25$ and maximal iteration $\{N\}_{-}\{i\}=500$;

3. Initialize the position of each fruit fly (X_axis,Y_axis);

4. Randomly allocate the direction and scope to the food source by each fruit fly according to the smell apparatus, which is denoted as following:

$\{X\}_{\_}\{i\}=X \backslash \_a x i s+r a n d o m \backslash$ value

14

$\{Y\} \_\{i\}=Y \backslash \_a x i s+r a n d o m \backslash$ value

15

1. Due to the accurate location information of the food source is fuzzy, the distance between ith fruit fly coordinate and original position $(0,0)$ is calculated first, described as \{dis\}_\{i\}. Then the proposed model parameter set $\{\backslash$ exists $\}$ _ $\{i\}$ can be obtained based on the $\{d i s\} \_\{i\}$ by calculating the following expressions:

$\{\text { dis }\}_{-}\{i\}=\backslash s q r t\left\{\{X\}_{-}\{i\}^{\wedge}\{2\}+\{Y\}_{-}\{i\}^{\wedge}\{2\}\right\}$

16

$\{\text { lexists }\}_{-}\{i\}=\backslash$ frac $\left.\{1\}\{\text { dis }\}_{-}\{i\}\right\}$ 
1. Replace the $\{\backslash \text { exists }\}_{-}\{i\}$ in objective function Fit $\backslash$ left(\{\exists $\left.\}_{-}\{i\} \backslash r i g h t\right)$ to calculate the smell concentration value $\{S\}_{-}\{i\}$ of ith fruit fly. Then the fruit fly with lowest $\{S\}_{-}\{i\}$ will be extracted out.

Finally, the $\{S\} \_\{i\}$ and corresponding position is recorded in the platform;

$\{S\}_{-}\{i\}=$ Fit $\backslash$ left $\left(\{\backslash \text { exists }\}_{-}\{i\} \backslash\right.$ right $)$

18

\left[optimal\_smell, optimal\_coordinate=min $\backslash$ left $\left(\{S\}_{-}\{i\} \backslash r i g h t\right) \backslash$ right $]$ 19

1. Obtained and recorded the optimal $\{S\}_{-}\{i\}$ and corresponding position. The fruit fly group flies towards the optimal point at the same time;

2. Repeat the steps 3-6. If latest value of the $\left\{S_{-}\right\}_{-}\{\}$is lower than the record, replacing it with lower result and keeping the original value in other cases; if the actual number of iterations arrives at the set value, i.e., $\{\mathrm{N}\} \_\{i\}=500$, stop the algorithm process. The principle of model parameter optimization by using FOA is shown in Fig. 6.

\subsection{Model Parameters Identification And Analysis}

So as to validate the effectiveness of the constructed model to capture the nonlinear hysteretic properties of the MR gel in the current dimension. The experimental data corresponding to the five current levels of $0 \mathrm{~A}, 0.2 \mathrm{~A}, 0.5 \mathrm{~A}, 0.8 \mathrm{~A}$ and $1 \mathrm{~A}$ under the strain amplitude and frequency of $10 \%$ and $0.1 \mathrm{~Hz}$ respectively are employed to identify the model parameters. Fig. 7-11 demonstrate the model tracking process in one data acquisition cycle under different current excitation, i.e. $0 \mathrm{~A}, 0.2 \mathrm{~A}, 0.5 \mathrm{~A}, 0.8 \mathrm{~A}$ and $1 \mathrm{~A}$ respectively. As illustrated from Fig. 7-11, it is obvious noted that the proposed model yields good agreements with hysteretic behaviors and strain stiffening of the MR gel. For instance, the nonlinear properties and strain stiffening phenomenon of the MR gel grows increasing prominent along with applied current. The test data and the predictive value shown in Fig. 8-11 express that the proposed model can perfectly fit the strain stiffening and nonlinearity caused by the ascending current.

In order to further investigate the efficient and ability of the constructed strain stiffening model to capture the nonlinear hysteretic loop of the MR gel, two typical model such as Bouc-Wen model and viscoelasticplastic model are utilized as comparison group. In the process of comparative study for the three parametric models, the RMSE is employed as a coefficient and the lower the value of the RMSE, the better the tracking capability of the model. The comparative study for the three model is conducted and the results are presented in Fig. 12-16. The corresponding RMSE values are listed in Tab.1. It is clearly seen from Fig. 12-16 that the proposed model has a superior performance to capture the nonlinearity and strain stiffening behavior of the MR gel compared with other two commonly model for the same experimental data, especially for the test dada corresponding to the higher current excitation, which can be clearly reflected in the Tab.1. 
Table 1

The comparison of the three models using RMSE

\begin{tabular}{|llll|}
\hline Current Level & Proposed model & Bouc-Wen model & Viscoelastic-plastic model \\
\hline OA & 1.049 & 2.912 & 3.631 \\
\hline $0.2 \mathrm{~A}$ & 1.468 & 8.420 & 7.723 \\
\hline 0.5A & 18.045 & 25.927 & 25.205 \\
\hline $0.8 \mathrm{~A}$ & 40.083 & 73.921 & 75.854 \\
\hline 1A & 48.629 & 78.734 & 93.153 \\
\hline
\end{tabular}

\subsection{Parameter Analysis Of The Proposed Model}

In order to better employ the constructed model to capture the nonlinear hysteretic behaviors and strain stiffening phenomenon of the MR gel and then realize the semi-active control in engineering application. The investigation of the influence of parameters on the proposed model is conducted, which is illustrated in Fig. 17. In this case, the experimental data gained from the test condition of the strain amplitude, frequency and current are $10 \%, 0.5 \mathrm{~Hz}$ and $0.5 \mathrm{~A}$ respectively to demonstrate the generality of the proposed model. The value of the model parameter corresponding to the above test condition are $\backslash \operatorname{text}\{c\}=126.59$, $\backslash$ text $\{k\}=2.39$, $\{\backslash$ alpha $\}=8.04, \backslash$ delta $=12.53, \epsilon=0.015$ and $\backslash$ beta $=-0.017$, which is set as reference group.

Figure 17a depicts five groups of the stress-strain responses in relation to various values of $\backslash$ text $\{c\}$ : $70.59,100.59,130.59,160.59$ and 190.59 . The main impact of $\backslash$ text $\{c\}$ on the stress-strain loop is to change the area, which is corresponding to the viscosity of the sample. It is clearly observed that the higher the value of $\backslash$ text $\{c\}$, the obvious the viscosity of the MR gel is achieved. Another phenomenon to be seen is that all the curve are integrated in the two points (highlighting with $\{L\}_{-}\{1\}$ and $\{L\}_{-}\{2\}$ ) at the maximum strain, i.e. $-10 \%$ and $10 \%$, which indicated that the $\backslash$ text $\{c\}$ has little influence on the elastic behavior for the sample.

The effect of the $\backslash$ text $\{k\}$ on the stress-strain loop is shown in Fig. 17b. Different from parameter $\backslash$ text $\{c\}$, the strain stiffening characteristics of the sample come into appear when the value of $\backslash$ text $\{k\}$ higher than 2.39. In addition, all the loops communicate two points, which are the zero strain position in the stressstrain curves. These two points are of great significance since the strain stiffening of the curve is gradually obvious after those two intersections.

Figure 17c expresses the hysteretic responses at different value of \{lalpha \}: 4.04, 6.04, 8.04, 10.04 and 12.04. Fig. 17d shows the five groups of the the stress-strain responses for the various of $\backslash$ delta, i.e. 8.53, $10.53,12.53,14.53$ and 16.53 . The influence of these two parameters on the stress-strain loop is somewhat similar. Two important points to be awarded are that: (1) the elastic characteristics of the model can be controlled by adjusting the value of \{lalpha $\}$ and \delta. Specifically, the higher the value of $\{\backslash$ alpha $\}$, the stronger the elastic response of the loop is obtained; however, the parameter $\{\backslash$ alpha $\}$ has 
opposite impact on the elasticity of the stress-strain loop. (2) For five groups of the loops for these two parameters, all the hysteretic loops intersect at two points and the strain stiffening phenomenon come into view in the Figure 17c and Fig. 17d after going through these two points clockwise.

Figure 17e presents the responses corresponding to the five values of the parameter $\epsilon: 0.013,0.014$, $0.015,0.016$ and 0.017 . The strain stiffening effect of the model is significant severe with this parameter compared with parameter \text $\{\mathrm{k}\}$. All the loops in Figure 17e can be divided into three regions according to the strain: from $-10 \%$ to $-5 \%,-5-5 \%$ and $5-10 \%$. The influence of parameter on the strain stiffening of the model is obvious in the strain range from $-10 \%$ to $-5 \%$ and $5-10 \%$, calling as mutation area. Especially at the two maximum strains, with the decrease of parameter value, the strain stiffening effect becomes more obviously. However, within the strain ranging from $-5-5 \%$, the parameter has little impact on the loop, which is called as transition area.

The effect of the parameter \beta on the stress-strain loop is depicted in Fig. 17f. It is obviously seen that the model description is linear-like loop for the parameter value of -0.015 , which is shown ellipse-like of the hysteretic loop. With the decreases of the parameter, the stress-strain loop gradually becomes nonlinear. Another vital point is noted that these five groups communicate in two points at the strain positions of $-5 \%$ and $5 \%$. After these two points, the nonlinear behavior of the model corresponding to different parameter values is gradually distinguished, which in detail makes the elastic characteristics become larger with the decrease of parameter values.

\subsection{Parameter Generalization}

To provide the theoretical foundation for the semi-active control of the MR gel and fulfill the engineering application, this section is focus on the model parameter generalization. The optimal values of the proposed model parameters in relative to the five current levels, i.e. $0 \mathrm{~A}, 0.2 \mathrm{~A}, 0.5 \mathrm{~A}, 0.8 \mathrm{~A}$ and $1 \mathrm{~A}$, are shown in Tab.2, which is obtained by the sinusoidal excitation with strain amplitude and frequency of $10 \%$ and $0.1 \mathrm{~Hz}$ respectively. Fig. 18 depicts the relationship between current excitation and all model parameters, i.e. $\backslash$ text $\{c\}, \backslash$ text $\{k\},\{\backslash a l p h a\}, \backslash d e l t a, \epsilon$ and $\backslash$ beta. It is clearly observed that the parameters such as $\backslash$ text $\{c\}, \backslash$ text $\{k\}$ and $\{\backslash a \mid p h a\}$ appear to change linearly with applied current. However, the relationship between the rest three model parameters, i.e. \delta, $\epsilon$ and $\backslash$ beta, and current is more complicate, which is expressed using a quadratic function. Therefore, the Polynomial fitting technology based on least square method is employed to describe those relationship and the following expressions is exhibited to show all the parameters as a function of the exciting current:

$\backslash \operatorname{text}\{c\}=123.8101 \mid+3.3654$

19

$\backslash$ text $\{k\}=-84.2251 \mid+2.3716$

20

$\{$ \alpha $\}=41.8452 \mathrm{I}-0.9771$

21

Page $11 / 28$ 
Idelta $=14.1099\{\mid\}^{\wedge}\{2\}-15.1958 \mid+5.6466$

22

$\epsilon=-0.4007\{\mid\}^{\wedge}\{2\}+0.5248 \mid-0.0876$

23

\beta $=0.3004\{\mid\}^{\wedge}\{2\}-0.3948 \mid+0.0477$

24

Table 2

The optimal value of the proposed model for various currents

\begin{tabular}{|lllllll|}
\hline Current & Itext\{c\} & Itext\{k\} & \{lalpha $\}$ & Idelta & $\boldsymbol{\epsilon}$ & Ibeta \\
\hline OA & 9.7661 & 0.1115 & 3.8131 & 5.6192 & -0.0911 & 0.0419 \\
\hline $0.2 \mathrm{~A}$ & 23.3854 & -13.7868 & 8.4151 & 3.0991 & 0.0094 & -0.0102 \\
\hline 0.5A & 58.9141 & -35.1826 & 18.8033 & 1.8564 & 0.0674 & -0.0729 \\
\hline 0.8A & 105.2695 & -67.8507 & 36.7389 & 2.2141 & 0.0792 & -0.0804 \\
\hline 1A & 129.0174 & -81.9963 & 43.7282 & 4.8022 & 0.0359 & -0.0401 \\
\hline
\end{tabular}

To verify the capability of the proposed model after reconstructed to capture the nonlinear hysteretic behaviors and strain stiffening phenomenon of the MR gel, the comparison study between experimental data corresponding to the applied currents of $0.6 \mathrm{~A}$ and $0.9 \mathrm{~A}$ and modeling results is conduced. Fig. 19 shows the predicting results of the proposed model after parameter generalization for the stresssampling time curve. It is clearly indicated that the generalization for the model parameters is reliability and effective since the tracking performance of the general model is perfect.

Therefore, the proposed general model can provide a theoretical basis for the semi-active control of MR gel in practical engineering applications.

\section{Conclusions}

In this paper, a novel parametric model is proposed to describe the high nonlinear hysteretic properties and obvious strain stiffening characteristics of MR gel. This model is composed of a dashpot, a hysteresis operator and a spring relating to tangent function, which are communicated in parallel mode with each other. To verify the validity of the model, the experimental data corresponding to the five current levels of $0 \mathrm{~A}, 0.2 \mathrm{~A}, 0.5 \mathrm{~A}, 0.8 \mathrm{~A}$ and $1 \mathrm{~A}$ under the strain amplitude and frequency of $10 \%$ and $0.1 \mathrm{~Hz}$ respectively are employed to identify the parameters. Fruit fly optimization algorithm (FOA) is employed to search the optimal solution of parameters. A comparison investigation with two typical model such as Bouc-Wen model and viscoelastic-plastic model is conduced to to evaluate the effectiveness of the developed model. The comparative research results indicate that the fitting stress by the novel model can perfectly track the test dada. The study of the parametric generalization for the proposed model shows the reliability and effective of the reconstructed model. Consequently, the proposed parametric model has 
a great performance to predict the nonlinear hysteretic behaviors and strain stiffening properties of MR gel and is applicable to design the controller to meet the requirement of the semi-active control in the engineering application.

\section{Declarations}

\section{Acknowledgments}

This research was supported by the National Natural Science Foundation of China (Grant Nos. 52075492, $51675485,51775510,11972323)$, the Zhejiang Provincial Natural Science Foundation of China (Grant Nos. LR18E050002, LR20A020002), Open Fund of Key Laboratory of Special Purpose Equipment and Advanced Processing Technology, Ministry of Education and Zhejiang Province (Grant No. 20201201002). This work was supported in part by Beijing Advanced Innovation Center for Intelligent Robots and Systems under Grant 2019 IRS07.

\section{Data availability}

The data generated in this research are available on the request from the corresponding authors.

\section{Conflict of interest}

The authors declare no conflict of interest.

\section{References}

[1] Imaduddin F, Mazlan S, Fatah, A, et al. Testing and parametric modeling of magnetorheological valve with meandering flow path [J]. NONLINEAR DYNAMICS, 2016, 85(1):287-302.

[2] Zhang G, Wang J. A novel phenomenological model for predicting the nonlinear hysteresis response of magnetorheological gel[J]. Materials \& design, 2020:109074.

[3] Wang L, Yu M, Fu J, et al. Investigation on the effects of doped dendritic Co particles on rheological property of magnetorheological gel[J]. Smart Materials and Structures, 2018, 27(10).

[4] Zhang G, Li Y, Yu Y, et al. Modeling the nonlinear rheological behavior of magnetorheological gel using a computationally efficient model [J]. Smart Materials and Structures, 2020, 29(10).

[5] Zhang G, Wang H, Wang J, et al. Dynamic rheological properties of polyurethane-based magnetorheological gels studied using oscillation shear tests[J]. RSC Advances, 2019, 9(18): 1012410134.

[6] Dehghani R; Khanlo H and Fakhraei J. Active chaos control of a heavy articulated vehicle equipped with magnetorheological dampers [J]. NONLINEAR DYNAMICS, 2017, 87(3):1923-1942. 
[7] Ge W, Brooker G, Woo J, et al. Magnetorheological Gel Mimicking Cervical Ripening as a Potential Model for Evaluating Shear Wave Elastography[J]. Ultrasound in Medicine \& Biology, 2020.

[8] Yu Y, Li Y, Li J, et al. A hysteresis model for dynamic behaviour of magnetorheological elastomer base isolator[J]. Smart Materials and Structures, 2016, 25(5): 055029.

[9] Zhang X , Li W, Gong X. An effective permeability model to predict field-dependent modulus of magnetorheological elastomers[J]. Communications in Nonlinear Science \& Numerical Simulation, 2008, 13(9):p.1910-1916.

[10] Jolly M, Carlson J, Muoz B . A model of the behaviour of magnetorheological materials[J]. Smart Materials \& Structures, 1996, 5(5):607-614(8).

[11] Peng J, Wang L, Lenci S, et al. Time-delay dynamics of the MR damper-cable system with one-to-one internal resonances [J]. NONLINEAR DYNAMICS, 2021, 105(2):1343-1356.

[12] Yu Y, Li Y, Li J. Nonparametric modeling of magnetorheological elastomer base isolator based on artificial neural network optimized by ant colony algorithm[J]. Journal of Intelligent Material Systems \& Structures, 2015, 26(14):1789-1798.

[13] Ismail M, Ikhouane F, Rodellar J, et al. The Hysteresis Bouc-Wen Model, a Survey[J]. Archives of Computational Methods in Engineering, 2009, 16(2): 161-188.

[14] Wen Y K 1976 Method of random vibration of hysteretic systems J. Eng. Mech. 102 249-63.

[15] Spencer J, Dyke S, Sain M, et al. Phenomenological model for magnetorheological dampers[J]. Journal of engineering mechanics, 1997, 123(3): 230-238.

[16] Kamath G, Wereley N. A nonlinear viscoelastic - plastic model for electrorheological fluids[J]. Smart Materials \& Structures, 1997, 6(3):351-359(9).

[17] Pan W. Mixed modified fruit fly optimization algorithm with general regression neural network to build oil and gold prices forecasting model[J]. Kybernetes: The International Journal of Systems \& Cybernetics, 2014, 43(7): 1053-1063.

[18] Kwok N, Ha Q, Nguyen T, et al. A novel hysteretic model for magnetorheological fluid dampers and parameter identification using particle swarm optimization[J]. Sensors \& Actuators A Physical, 2006, 132(2):441-451.

[19] Jie F, Junjie L, Guanyao L, et al. Genetic algorithm based nonlinear self-tuning fuzzy control for timevarying sinusoidal vibration of a magnetorheological elastomer vibration isolation system[J]. Smart Materials and Structures, 2018, 27(8):085010-. 
[20] Pan W. A new fruit fly optimization algorithm: taking the financial distress model as an example[J], Knowledge-based System, 2012, 26(2): 69-74.

[21] Yu Y, Li Y, Li J, et al. Self-adaptive step fruit fly algorithm optimized support vector regression model for dynamic response prediction of magnetorheological elastomer base isolator[J]. Neurocomputing, 2016, 211(oct.26):41-52.

[22] Yu Y , Li J , Li Y , et al. Comparative investigation of phenomenological modeling for hysteresis responses of magnetorheological elastomer devices[J]. International Journal of Molecular ences, 2019, 20(13):3216.

[23] Kwok N, Ha Q, Nguyen M, et al. Bouc-Wen model parameter identification for a MR fluid damper using computationally efficient GA[J]. ISA transactions, 2007, 46(2): 167-179.

[24] Kamath G, Wereley N. A nonlinear viscoelastic - plastic model for electrorheological fluids[J]. Smart Materials \& Structures, 1997, 6(3):351-359(9).

[25] Pan W. Using modified fruit fly optimisation algorithm to perform the function test and case studies[J]. Connection ence, 2013, 25(2-3):151-160.

\section{Figures}
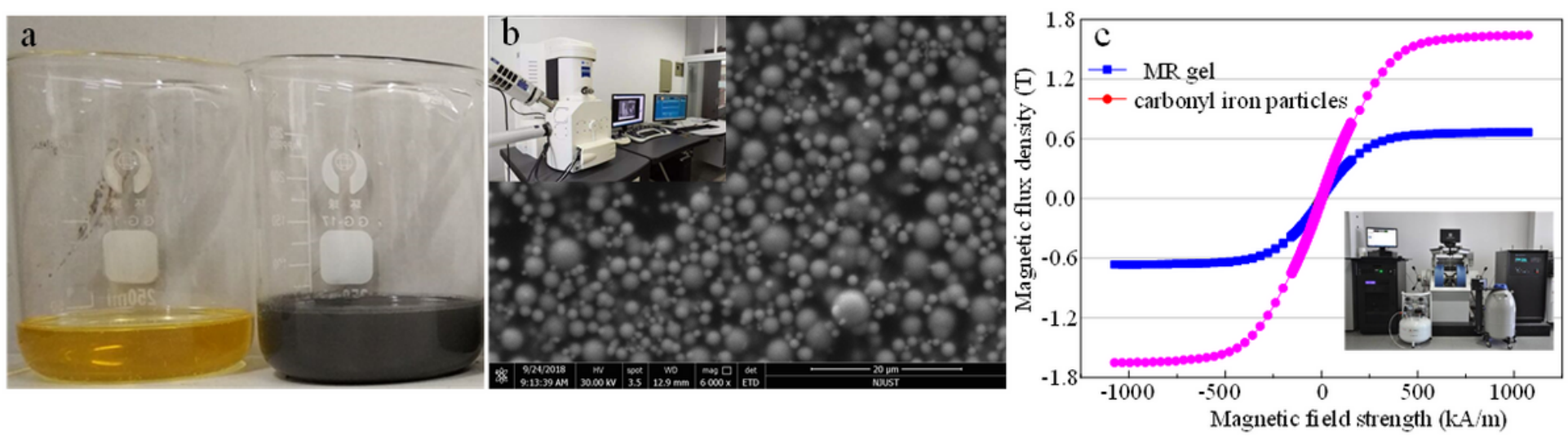

\section{Figure 1}

Developed MR gel and physical properties. (a) MR gel and its matrix; (c) microstructure of the MR gel and (c) magnetic property of the CIP and MR gel 

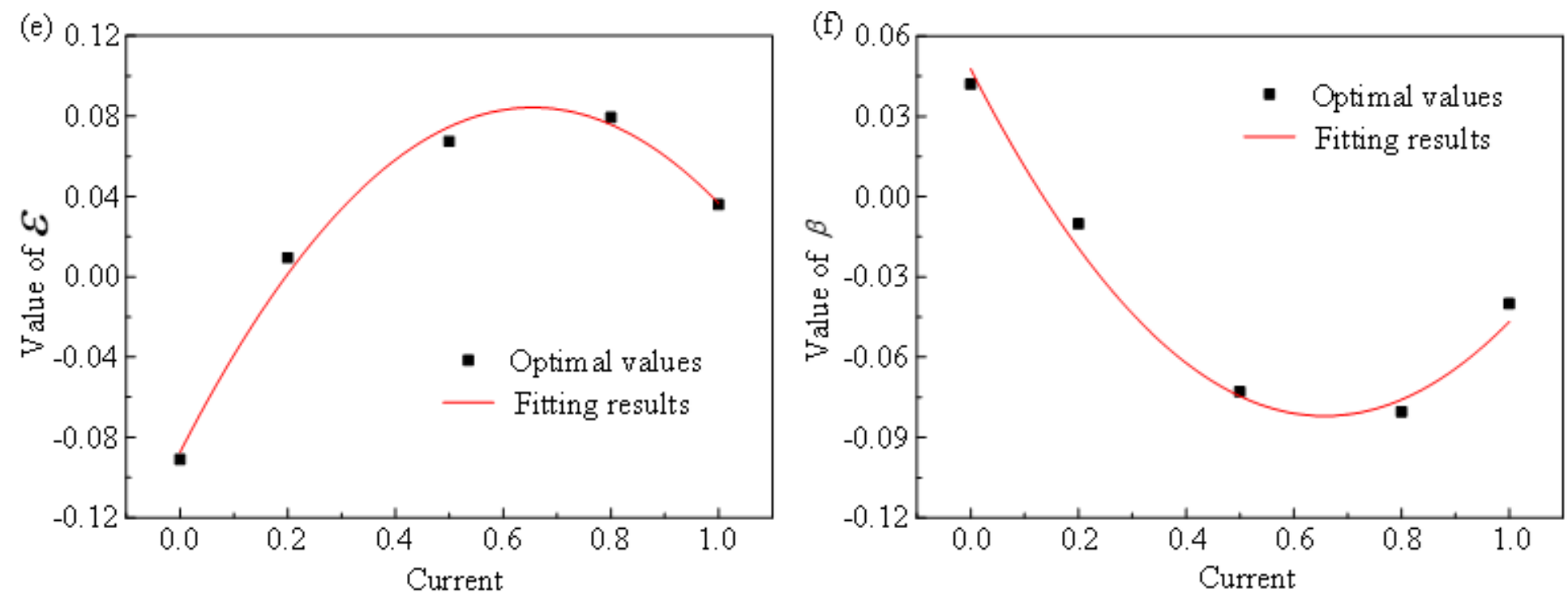

Figure 2

(a) The test device and principle; (b) the condition of the LAOS and (c) dynamic responses of the MR gel

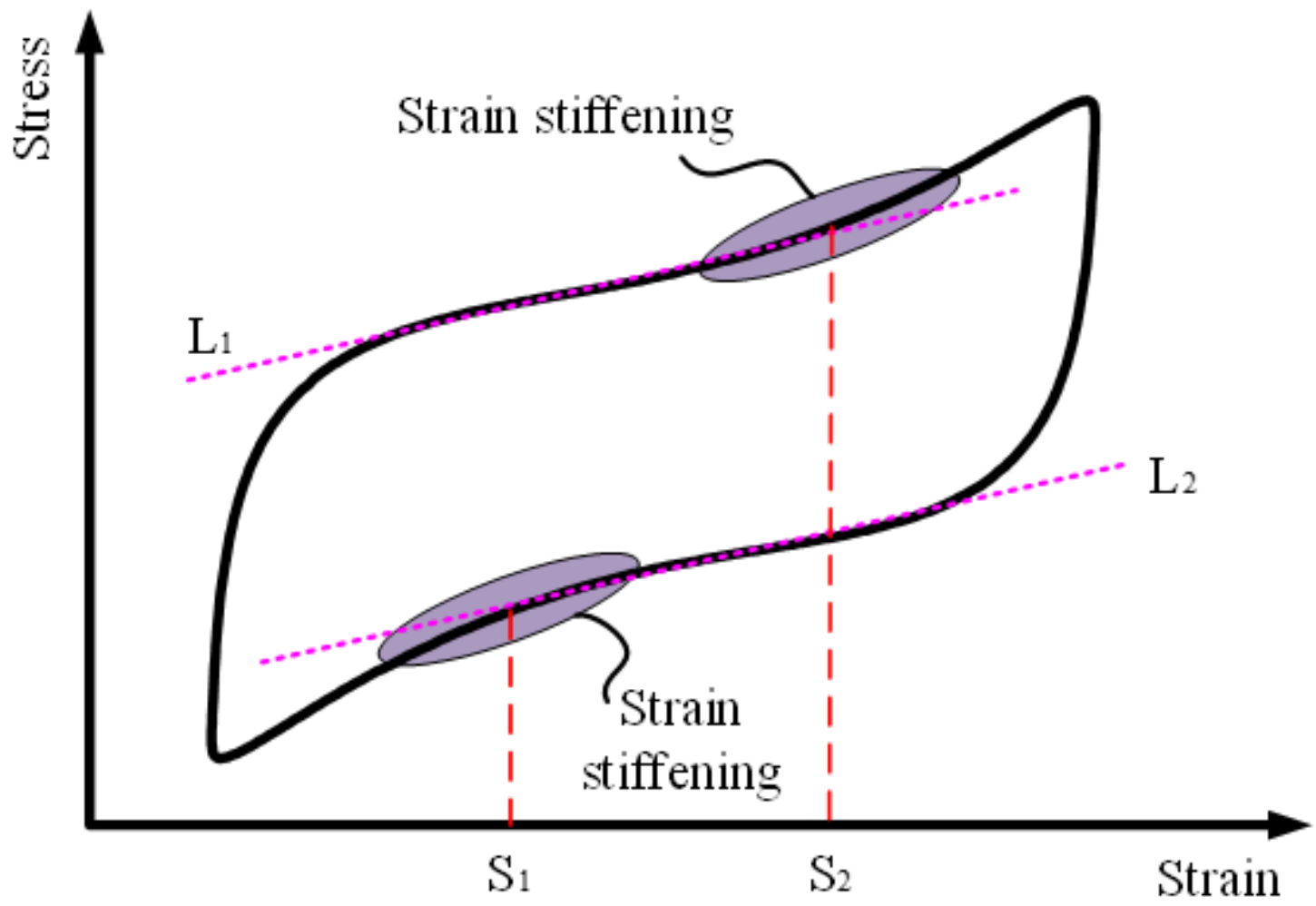

Figure 3

Strain stiffening phenomenon (current: 1A) 


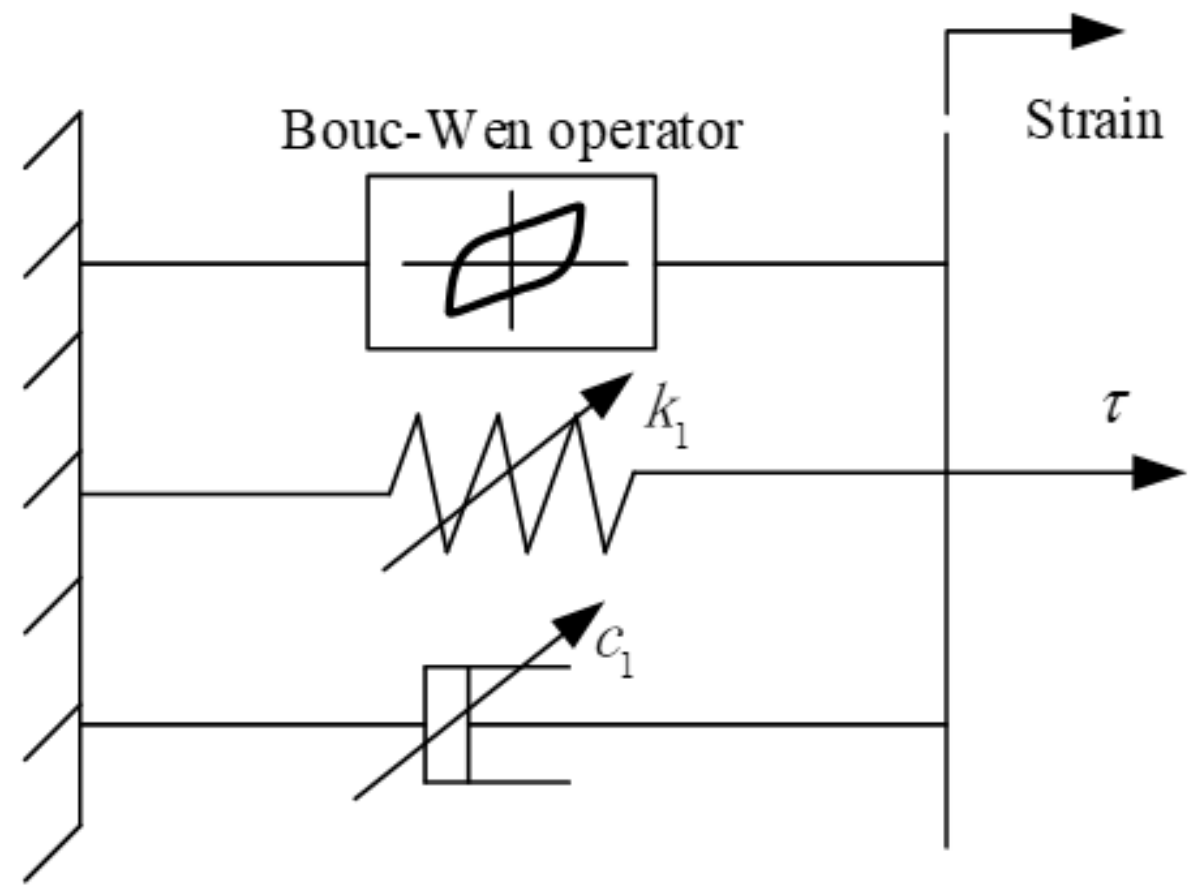

Figure 4

Bouc-Wen model 


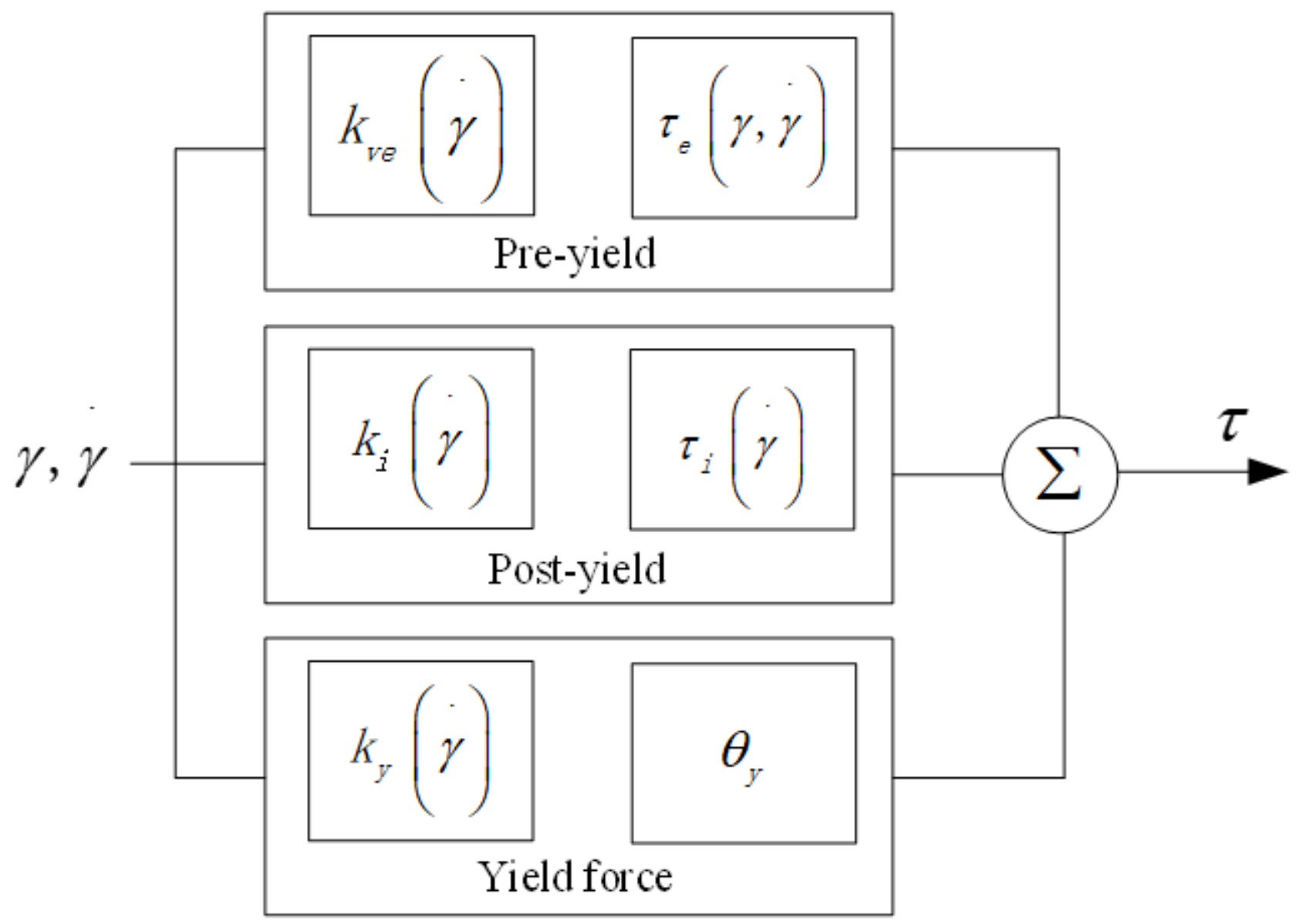

Figure 5

Viscoelastic-plastic model

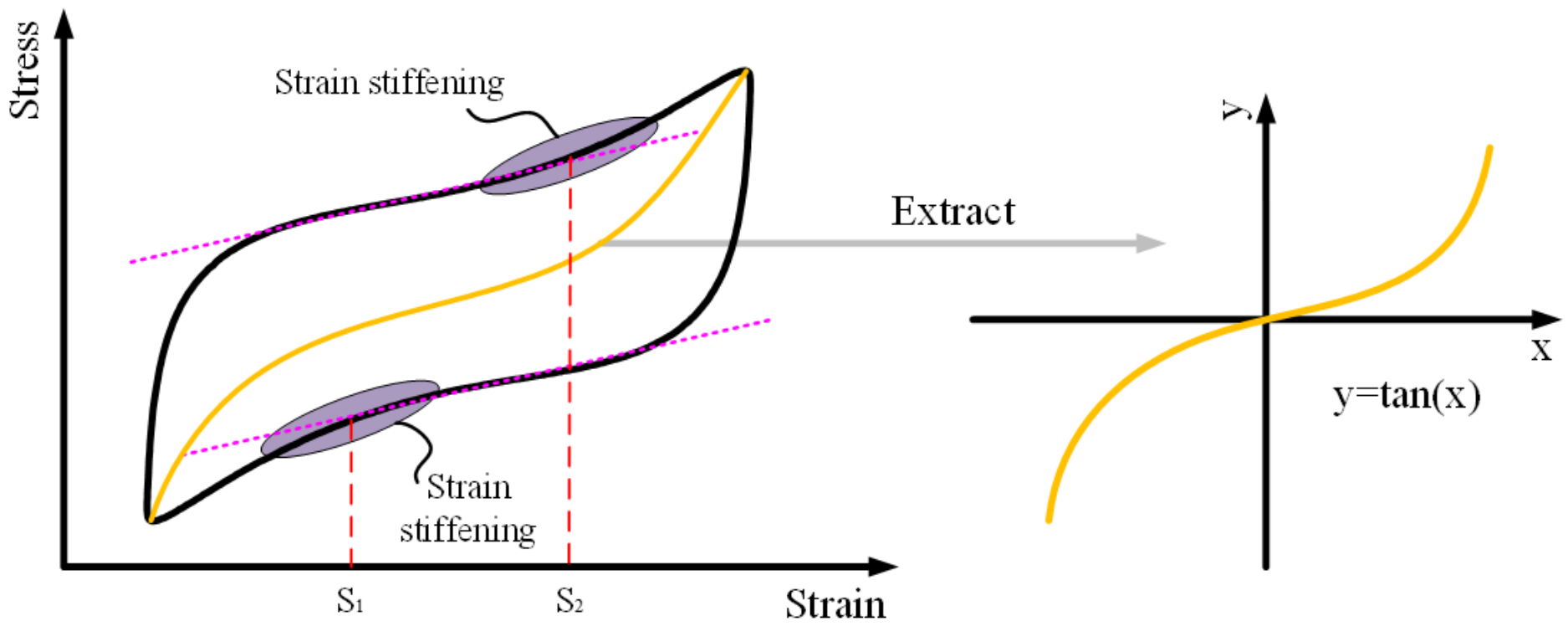


Figure 6

Modeling principle

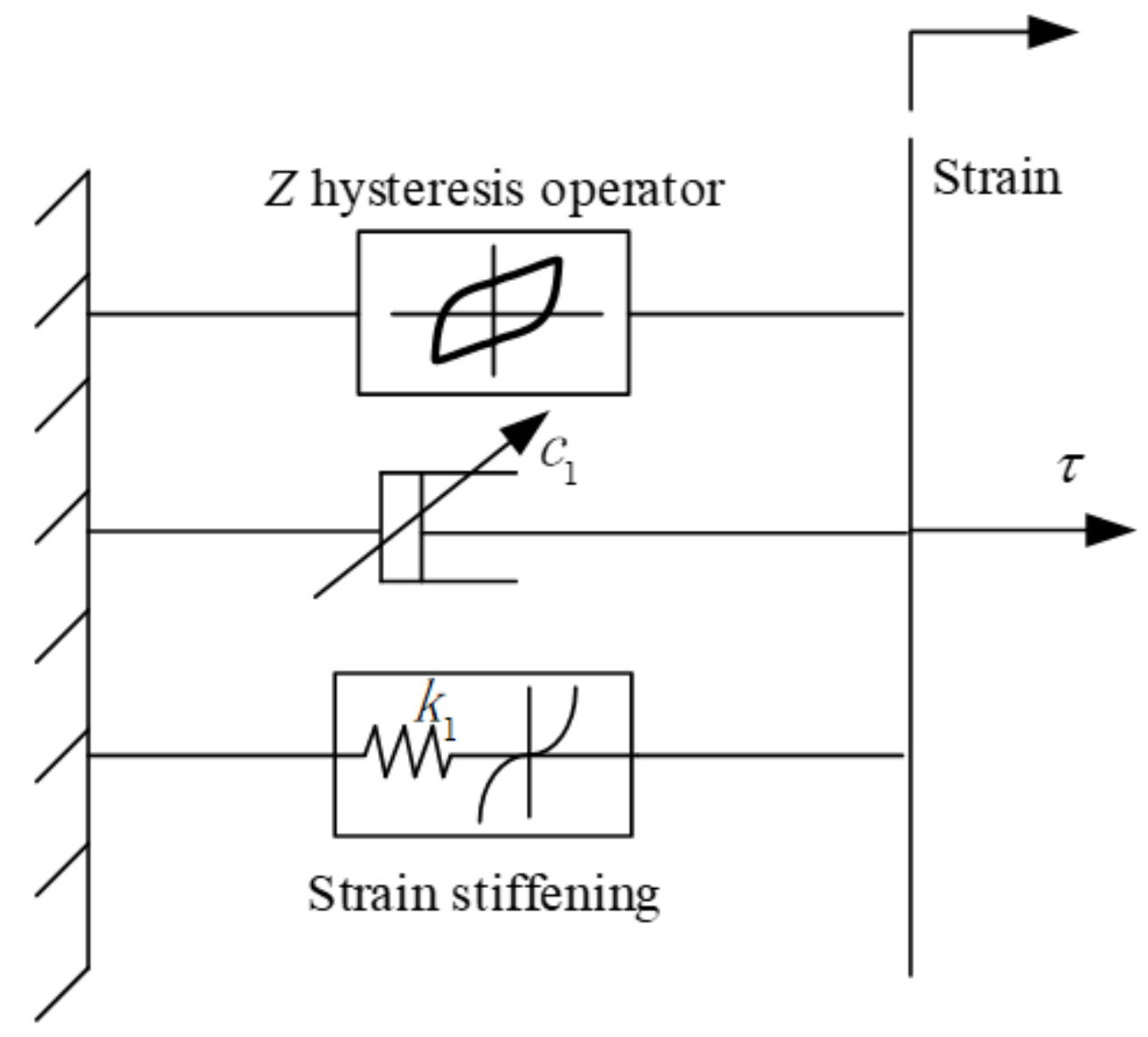

Figure 7

The proposed parametric model 


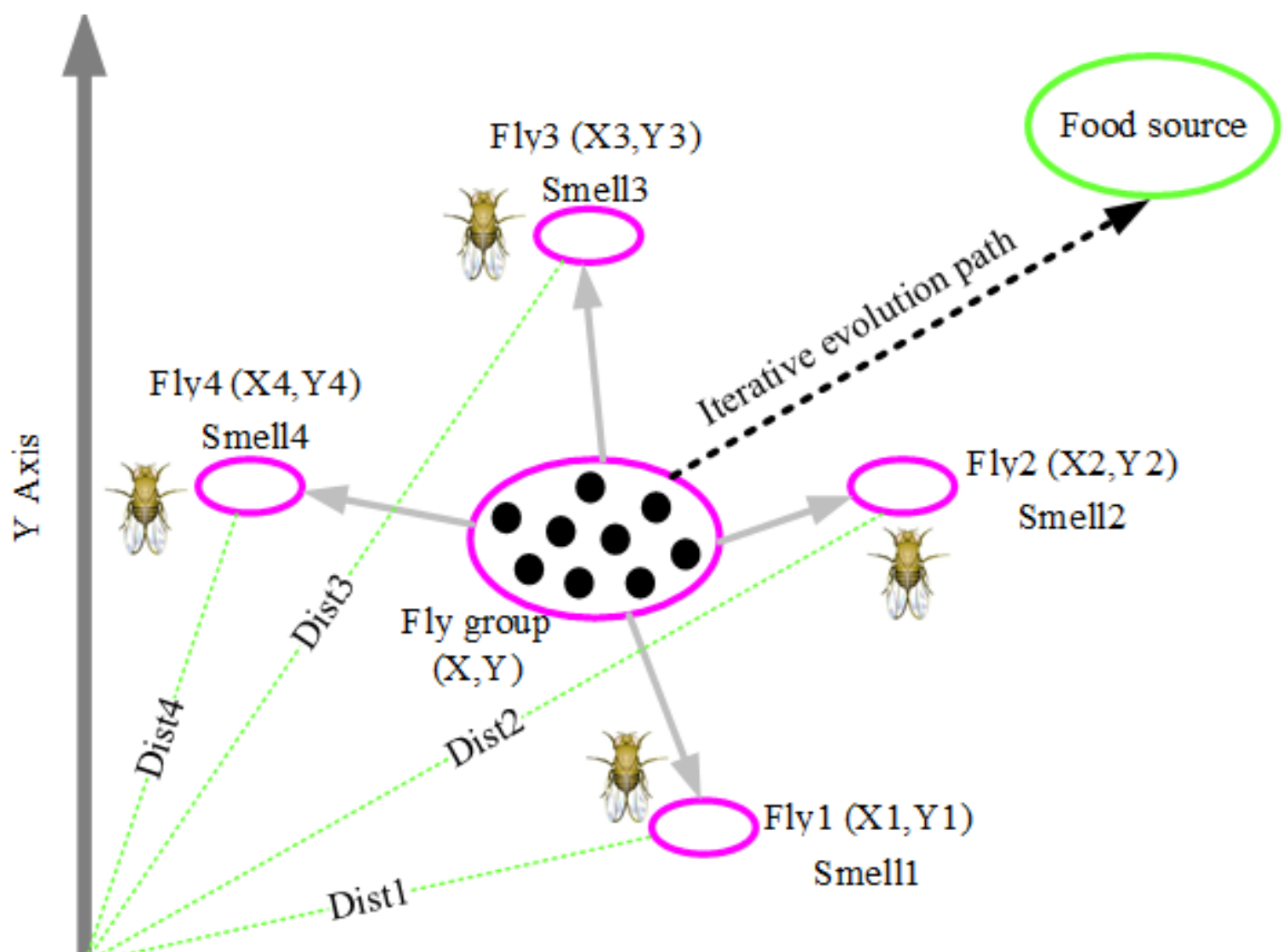

$\mathrm{XAx}$ is

Figure 8

The iteration evolution path of the fruit fly swarm to food source

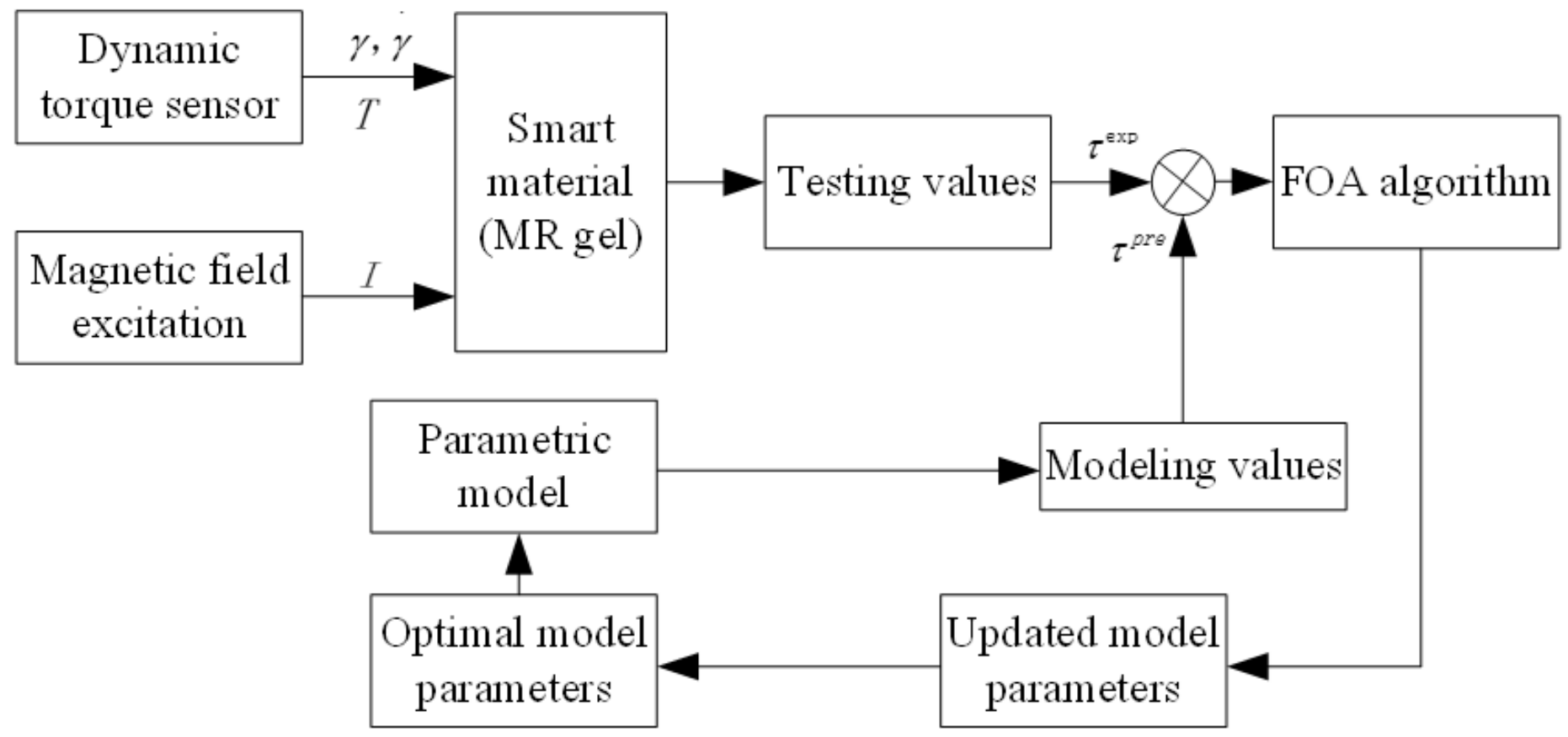




\section{Figure 9}

The principle of model parameter optimization
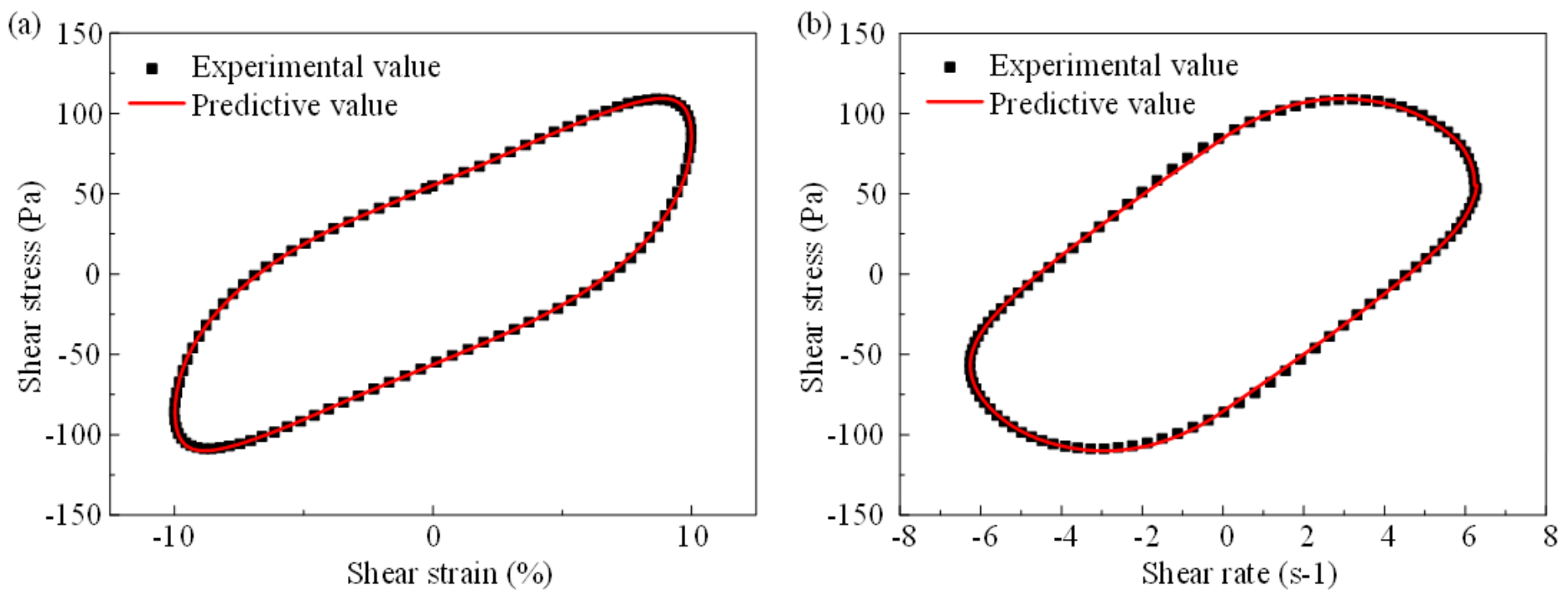

\section{Figure 10}

Comparison between experimental value and predictive result of the proposed model at the current level of 0A. (a) Stress-strain; (b) Stress-rate
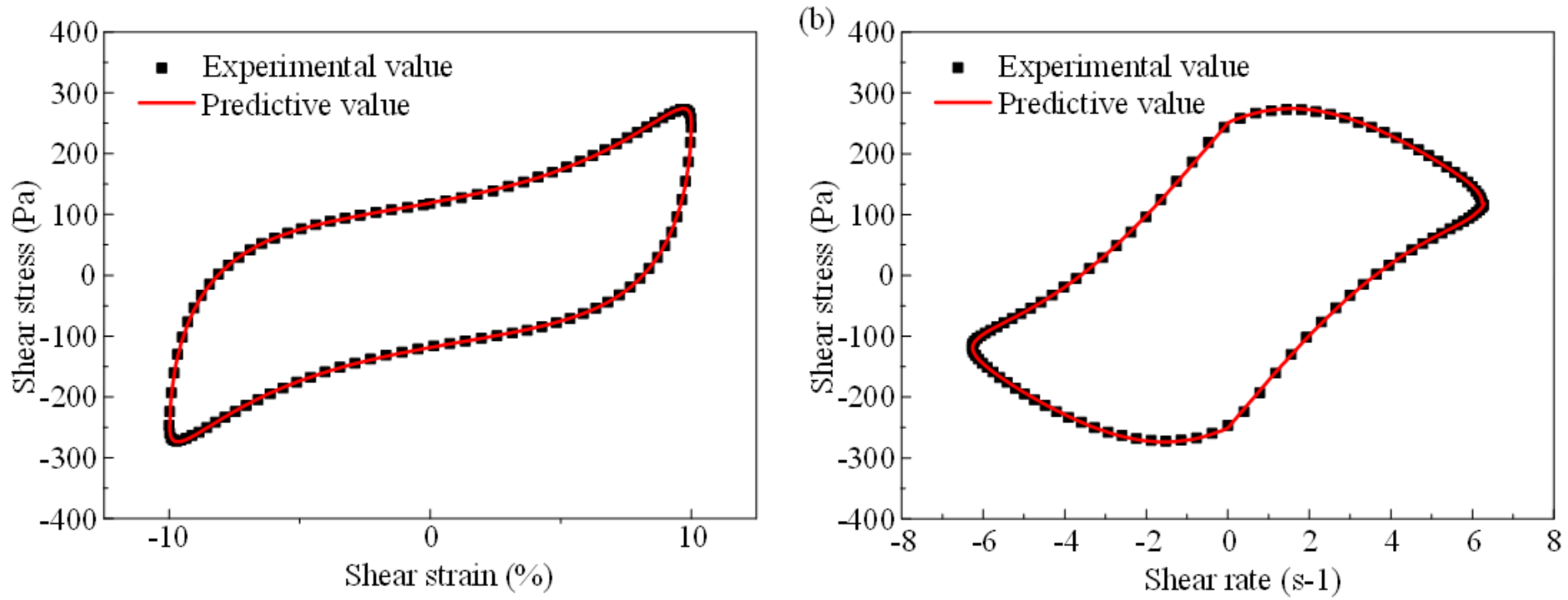

\section{Figure 11}

Comparison between experimental value and predictive result of the proposed model at the current level of 0.2A. (a) Stress-strain; (b) Stress-rate 

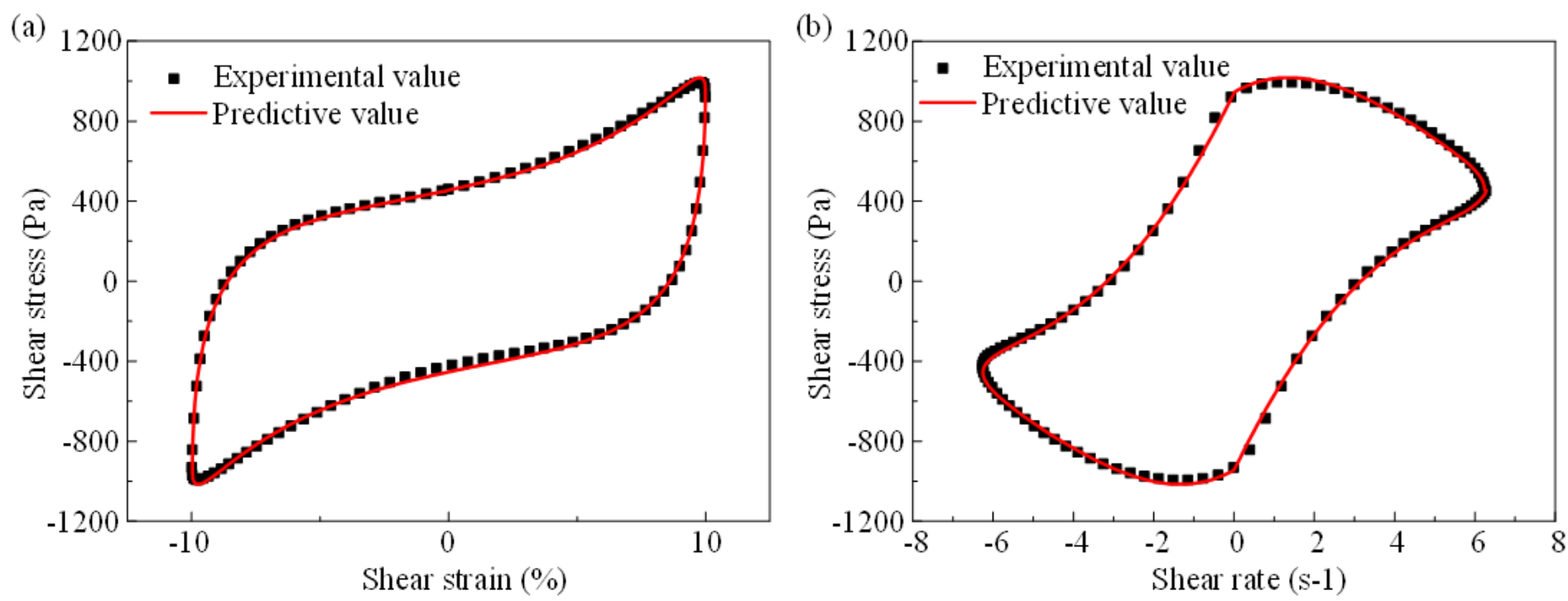

Figure 12

Comparison between experimental value and predictive result of the proposed model at the current level of 0.5A. (a) Stress-strain; (b) Stress-rate
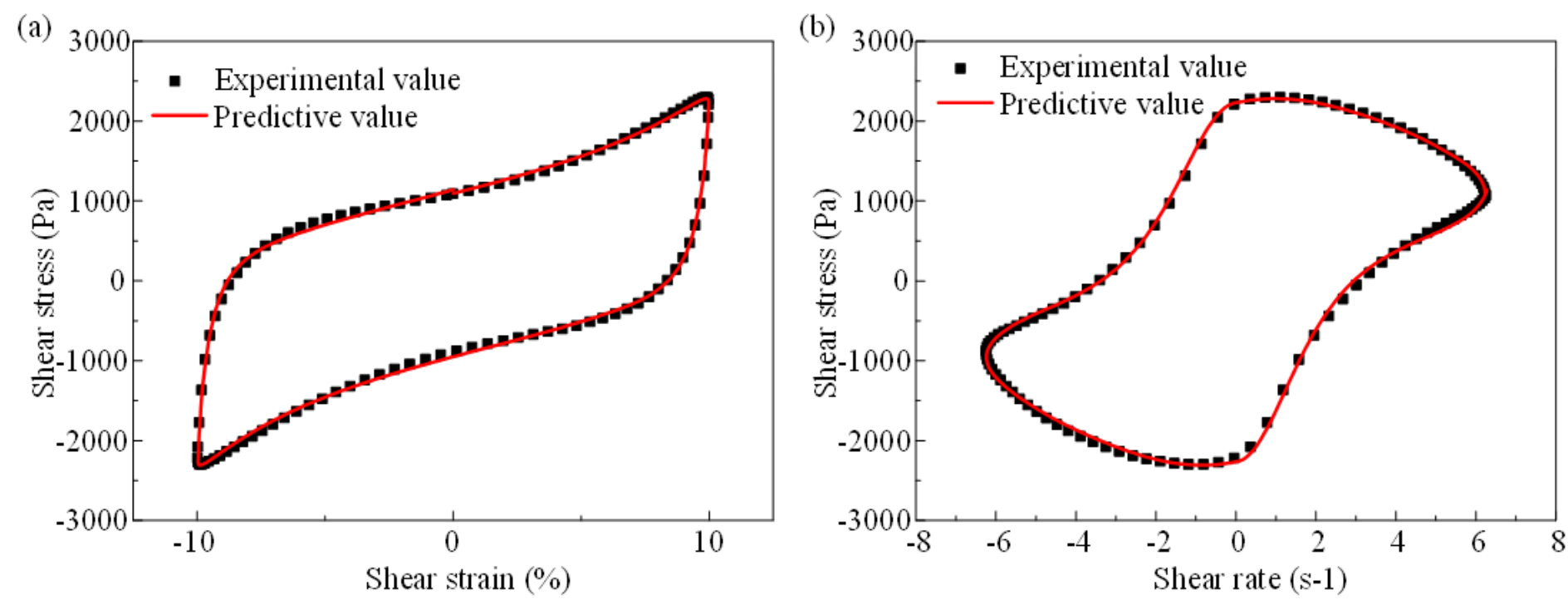

Figure 13

Comparison between experimental value and predictive result of the proposed model at the current level of 0.8A. (a) Stress-strain; (b) Stress-rate 

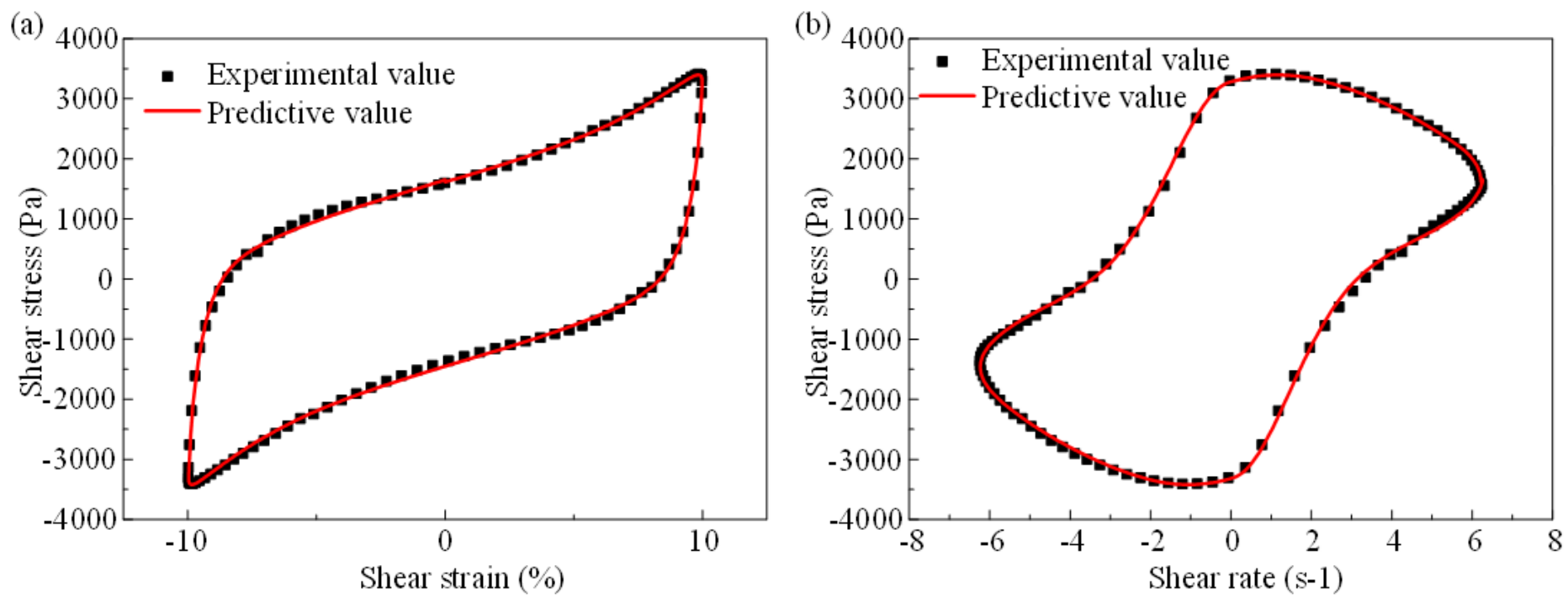

\section{Figure 14}

Comparison between experimental value and predictive result of the proposed model at the current level of 1A. (a) Stress-strain; (b) Stress-rate
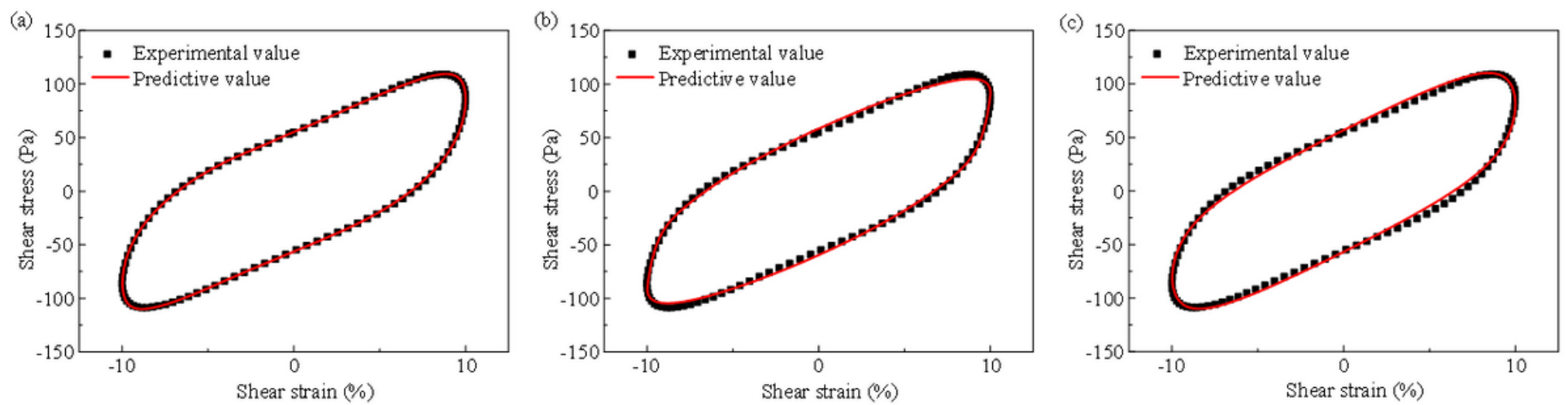

\section{Figure 15}

The predictability of the proposed model and two classical models. (a) Proposed model; (b) Bouc-Wen model and (c) Viscoelastic-plastic model; at the current level of $0 \mathrm{~A}$ 

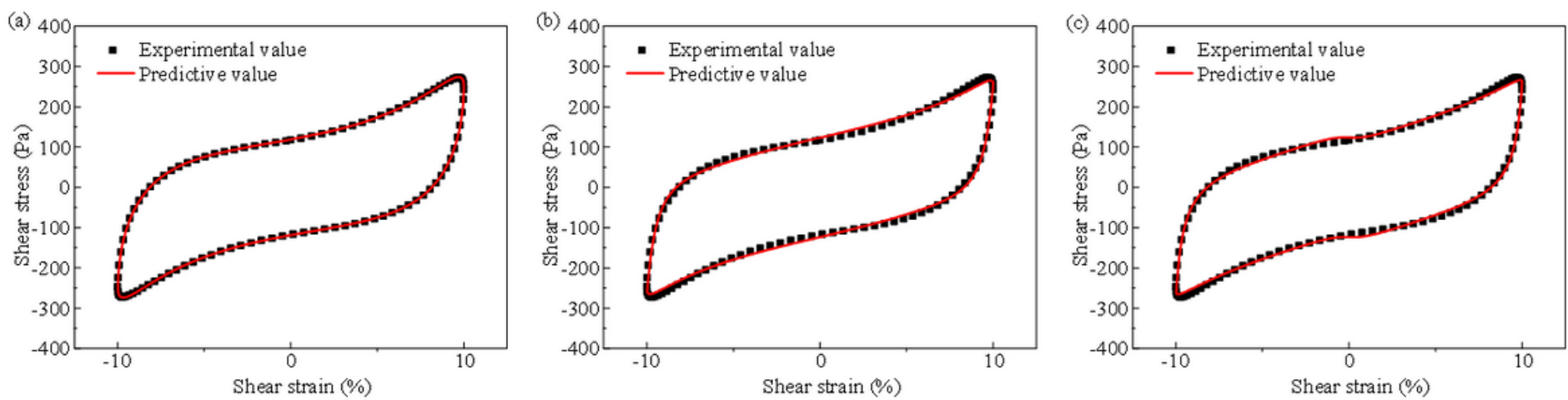

Figure 16

The predictability of the proposed model and two classical models. (a) Proposed model; (b) Bouc-Wen model and (c) Viscoelastic-plastic model; at the current level of $0.2 \mathrm{~A}$
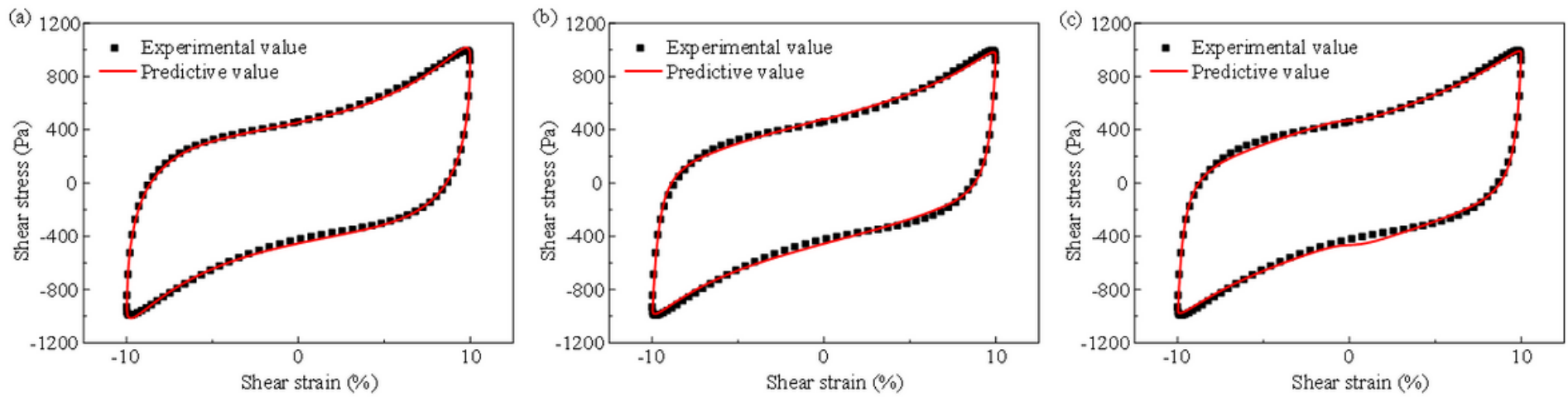

\section{Figure 17}

The predictability of the proposed model and two classical models. (a) Proposed model; (b) Bouc-Wen model and (c) Viscoelastic-plastic model; at the current level of $0.5 \mathrm{~A}$
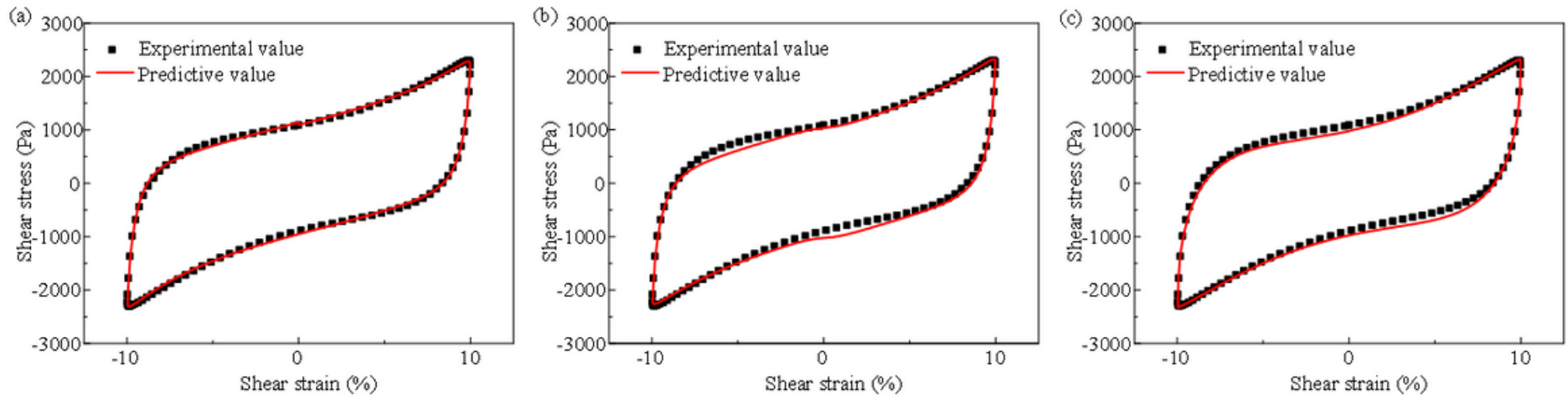

Figure 18 
The predictability of the proposed model and two classical models. (a) Proposed model; (b) Bouc-Wen model and (c) Viscoelastic-plastic model; at the current level of $0.8 \mathrm{~A}$
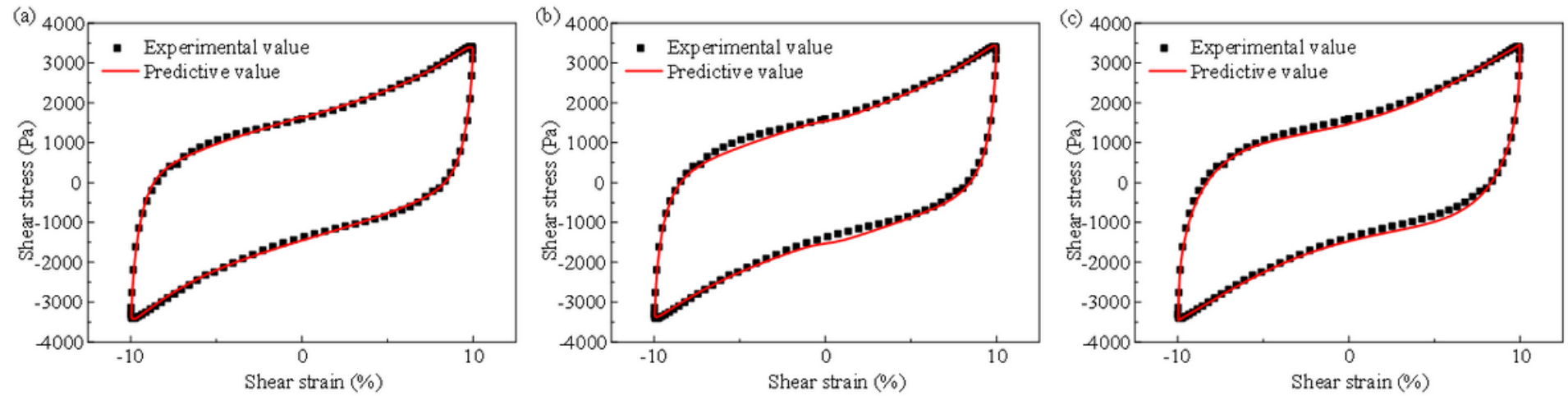

\section{Figure 19}

The predictability of the proposed model and two classical models. (a) Proposed model; (b) Bouc-Wen model and (c) Viscoelastic-plastic model; at the current level of $1 \mathrm{~A}$ 

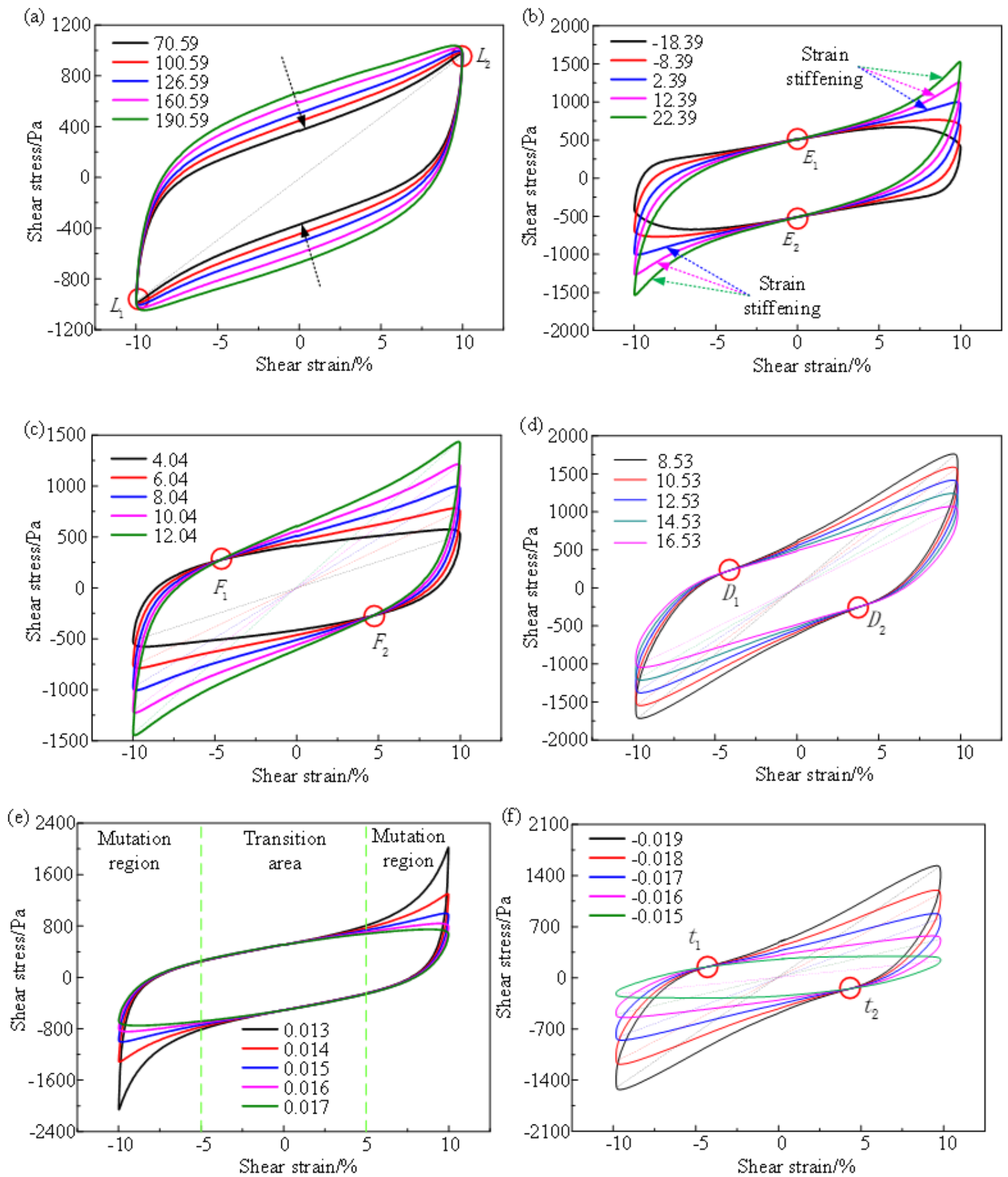

\section{Figure 20}

The influence of the input parametric values on the proposed model output. (a) c; (b) k; (c) k; (d) $\delta$; (e) $\varepsilon$ and (f) $\beta$. 

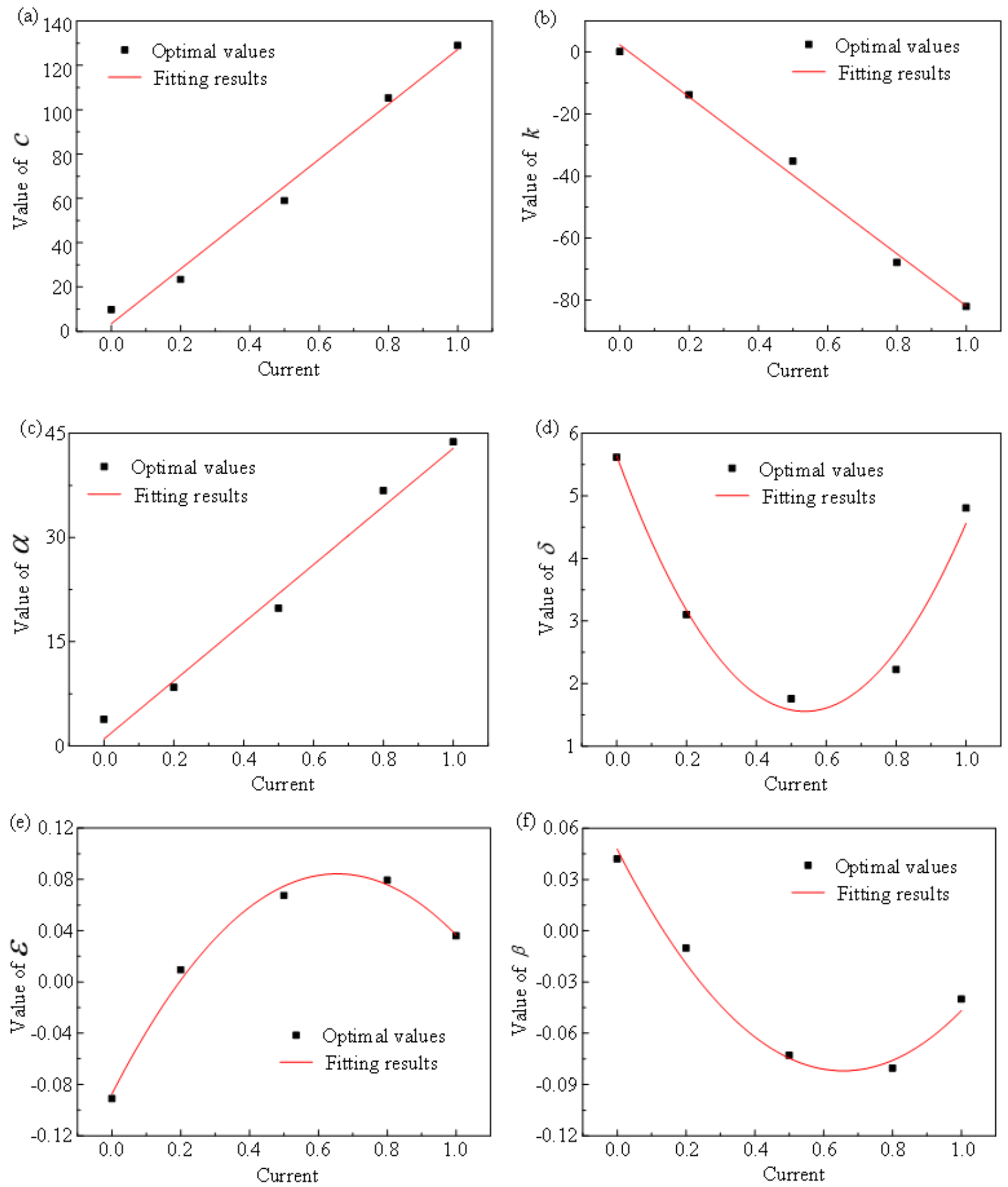

\section{Figure 21}

The relationship between the currents and the model parameters. 

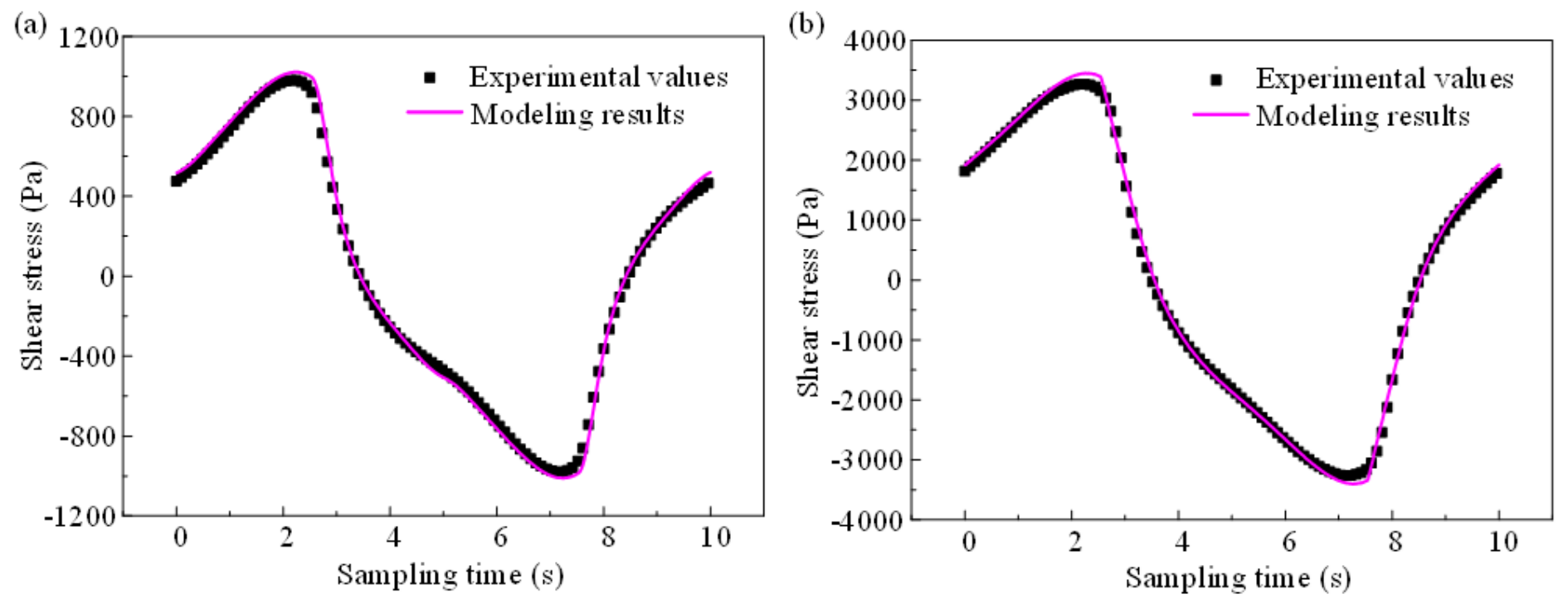

\section{Figure 22}

The predicting results of the proposed model after parameter generalization. Current: (a) 0.6A and (b) $0.9 \mathrm{~A}$ 